Pacific

Journal of

Mathematics

\title{
POINCARÉ-EINSTEIN METRICS AND THE SCHOUTEN TENSOR
}

\author{
Rafe Mazzeo and Frank Pacard
}




\title{
POINCARÉ-EINSTEIN METRICS AND THE SCHOUTEN TENSOR
}

\author{
Rafe Mazzeo and Frank PaCARd
}

\begin{abstract}
We examine the space of conformally compact metrics $g$ on the interior of a compact manifold with boundary which have the property that the $k^{\text {th }}$ elementary symmetric function of the Schouten tensor $A_{g}$ is constant. When $k=1$ this is equivalent to the familiar Yamabe problem, and the corresponding metrics are complete with constant negative scalar curvature. We show for every $k$ that the deformation theory for this problem is unobstructed, so in particular the set of conformal classes containing a solution of any one of these equations is open in the space of all conformal classes. We then observe that the common intersection of these solution spaces coincides with the space of conformally compact Einstein metrics, and hence this space is a finite intersection of closed analytic submanifolds.
\end{abstract}

Let $\bar{M}^{n+1}$ be a smooth compact manifold with boundary. A metric $g$ defined on its interior is said to be conformally compact if there is a nonnegative defining function $\rho$ for $\partial \bar{M}$ (i.e., $\rho=0$ only on $\partial \bar{M}$ and $d \rho \neq 0$ there) such that $\bar{g}=\rho^{2} g$ is a nondegenerate metric on $\bar{M}$. The precise regularity of $\rho$ and $\bar{g}$ is somewhat peripheral and shall be discussed later. Such a metric is automatically complete. Metrics which are conformally compact and also Einstein are of great current interest in (some parts of) the physics community, since they serve as the basis of the AdS/CFT correspondence [24], and they are also quite interesting as geometric objects. Since they are natural generalizations of the hyperbolic metric on the ball $B^{n+1}$, as well as the complete constant negative Gauss curvature metrics on hyperbolic Riemann surfaces - which exist in particular on the interiors of arbitrary smooth surfaces with boundary - and which are often called Poincaré metrics [19], we say that a metric which is both conformally compact and Einstein is Poincaré-Einstein (or P-E for short). Until recently, beyond a handful of examples, the only general existence result concerning the existence of P-E metrics was the local perturbation theory of Graham and Lee [11], which gives an infinite dimensional family of such metrics in a neighborhood of the hyperbolic metric on the ball, parametrized by conformal classes on the boundary sphere near to the standard one. Recently 
many new existence results have been obtained, including further perturbation results by Biquard [7] and Lee [13], and Anderson has some important global existence results in dimension four [3]. Many interesting geometric and topological properties of these metrics have also been found [10], [23], $[\mathbf{1}]$ and $[\mathbf{2}]$; this last paper also surveys a number of intriguing examples of P-E metrics.

A common thread through the analytic approaches to the construction of these metrics is the possible existence of an $L^{2}$ obstruction, or more simply a finite dimensional cokernel of the (suitably gauged) linearization of the Einstein equations around a solution. For any P-E metric where this obstruction is trivial, the implicit function theorem readily implies that the moduli space $\mathcal{E}$ of $\mathrm{P}$-E metrics is (locally) a Banach manifold, parametrized by conformal classes of metrics on $\partial \bar{M}$. (Actually, the smoothness of $\mathcal{E}$ is true in generality [3], but this geometric parametrization breaks down.) Unfortunately, the only known geometric criteria ensuring the vanishing of this obstruction are strong global ones [13].

One purpose of this note is to introduce some new ideas into this picture which may help elucidate the structure of this moduli space. We consider a related family of conformally compact metrics which satisfy certain scalar nonlinear equations, including and generalizing the familiar Yamabe equation, which we introduce below. These are sometimes called the $\sigma_{k}$-Yamabe equations, $k=1, \cdots, n+1$. The hyperbolic metric on the ball, or indeed an arbitrary P-E metric on any manifold with boundary satisfies each of these equations, and conversely, in this particular (conformally compact) setting, metrics which satisfy every one of these scalar problems are also P-E. The punchline is that, in the conformally compact case, the deformation theory for the $\sigma_{k}$-Yamabe equations is always unobstructed! This fact seems to have been unappreciated, except for the case $k=1$. The full implications of this statement in the conformally compact case for the moduli space of $\mathrm{P}-\mathrm{E}$ metrics is not completely evident at this point, but this relationship seems quite likely to be of some value. Furthermore, the deformation theory for these $\sigma_{k}$-Yamabe metrics is new, and also of some interest.

To define these equations, recall the Schouten tensor $A_{g}$, defined for any metric $g$ on a manifold of dimension $n+1$ by the formula

$$
A_{g}:=\frac{1}{n-1}\left(\operatorname{Ric}-\frac{R}{2 n} g\right)
$$

here Ric $:=\operatorname{Ric}_{g}$ and $R:=R_{g}$ are the Ricci tensor and scalar curvature function for $g$. This tensor occupies a prominent position in conformal geometry because it transforms quite nicely under conformal changes of 
metric. In fact, if $\widetilde{g}:=e^{2 u} g$, then

$$
A_{\widetilde{g}}=A_{g}-D d u+d u \otimes d u-\frac{1}{2}|d u|^{2} g .
$$

We refer to $[\mathbf{6}]$ for a derivation of this formula. For later reference, note that in terms of any local coordinate system $w$,

$$
\begin{aligned}
|d u|^{2} g & :=\sum_{i, j, k, \ell} g^{k \ell} \partial_{w_{k}} u \partial_{w_{\ell}} u g_{i j} d w^{i} d w^{j}, \\
d u \otimes d u & :=\sum_{i, j} \partial_{w_{i}} u \partial_{w_{j}} u d w^{i} d w^{j},
\end{aligned}
$$

and

$$
D d u:=\sum_{i, j}\left(\partial_{w_{i} w_{j}}^{2} u-\sum_{k} \Gamma_{i j}^{k} \partial_{w_{k}} u\right) d w^{i} d w^{j} .
$$

We have (somewhat inconsistently) used raised indices in the differentials (i.e., $d w^{i}$, etc.) in accord with the standard summation convention.

Definition 1. The metric $g$ is a $\sigma_{k}$-Yamabe metric if $\sigma_{k}\left(A_{g}\right)$, the $k^{\text {th }}$ elementary symmetric function of the eigenvalues of $A_{g}$ computed with respect to $g$, is constant.

The problem of finding $\sigma_{k}$-Yamabe metrics is usually posed as a problem in conformal geometry: Starting with an arbitrary metric $g$ and given $\beta \in \mathbb{R}$, the $\sigma_{k}$-Yamabe problem consists in finding a new metric $\widetilde{g}=e^{2 u} g$, in the conformal class of $g$, such that $\sigma_{k}\left(A_{\widetilde{g}}\right)=(-1)^{k} \beta$. (In the main case of interest here, the eigenvalues of $A_{g}$ are all negative, and so the constant $\beta$ is positive; this explains our choice of sign.) This way we reduce the problem to finding a solution $u$ to some scalar nonlinear partial differential equation. Notice that when $k=1, \sigma_{1}\left(A_{g}\right)=R / 2 n$, and so $\widetilde{g}$ is a $\sigma_{1}$-Yamabe metric if and only if its scalar curvature is constant. In this case the equation for $u$ becomes

$$
\Delta_{g} u+\frac{n-1}{2}\left|\nabla_{g} u\right|^{2}-\frac{R}{2 n}=\beta e^{2 u} .
$$

Defining $v$ by $v^{4 /(n-1)}=e^{2 u}$ (and keeping in mind that $\operatorname{dim} M=n+1$ ), the equation for $v$ assumes the familiar form

$$
\Delta_{g} v-\frac{R(n-1)}{4 n} v=-\frac{n-1}{2} \beta v^{\frac{n+3}{n-1}},
$$

and the existence theory when $M$ is compact is complete and by now wellknown [14]. However, when $k>1$, the equation for $u$ is fully nonlinear and the existence theory is much less well understood. Recent significant progress has been made by Chang-Gursky-Yang [8] when $k=2$, and also by Viaclovsky [22], but much remains to be understood. In particular, 
in contrast with the ordinary Yamabe problem, for $k>1$ the $\sigma_{k}$-Yamabe problem seems to be somewhat more tractable for positively curved metrics: a crucial a priori $\mathcal{C}^{2}$ estimate is missing in the case where all eigenvalues of $A_{g}$ are negative [22].

We now write out the $\sigma_{k}$-Yamabe equations (within a conformal class) more explicitly. Fixing $g$ and using (2), we see that

$$
\widetilde{g}=e^{2 u} g \quad \text { satisfies } \quad \sigma_{k}\left(A_{\widetilde{g}}\right)=(-1)^{k} \beta
$$

provided

$$
\mathcal{F}_{k}(g, u, \beta):=\sigma_{k}\left(D d u-d u \otimes d u+\frac{1}{2}|d u|^{2} g-A_{g}\right)-\beta e^{2 k u}=0 .
$$

The symmetric function of the eigenvalues of $A_{\widetilde{g}}$ here is computed with respect to $g$ rather than $\widetilde{g}$, which accounts for the exponential factor; the sign on the final term comes from taking $\sigma_{k}$ of $-A_{\widetilde{g}}$. For any constant $\beta$, we define

$$
\Sigma_{k}(\beta):=\left\{\widetilde{g}=e^{2 u} g: \quad \mathcal{F}_{k}(g, u, \beta)=0\right\},
$$

which is some subset within the space of all metrics on $M$.

As already indicated, the main result here involves the perturbation theory for solutions of $\mathcal{F}_{k}(g, u, \beta)=0$, or equivalently, the structure of the sets $\Sigma_{k}(\beta)$, in the case where $M^{n+1}$ is a manifold with boundary and all metrics are conformally compact. In this case, we will fix a defining function $\rho$ for $\partial \bar{M}$ and write any conformally compact metric $g$ on $M$ as $g=\rho^{-2} \bar{g}$ where $\bar{g}$ is a metric on $\bar{M}$.

Since conformally compact metrics have asymptotically negative (in fact, isotropic) sectional curvatures, $\Sigma_{k}(\beta)$ is nonempty only when $\beta>0$. Indeed, a brief calculation shows that when $g=\rho^{-2} \bar{g}$, then near any point of the boundary (where $\rho=0$ ),

$$
A_{g}=-\frac{1}{2}|d \rho| \frac{2}{g} g+\mathcal{O}\left(\rho^{-1}\right) .
$$

Notice that although $g$ only determines $\rho$ and $\bar{g}$ up to a conformal factor (i.e., $g$ is also equal to $(a \rho)^{-2}\left(a^{2} \bar{g}\right)$ for any $\left.a \in \mathcal{C}(\bar{M})\right)$, the function $|d \rho| \frac{2}{g}$ is welldefined at $\rho=0$, regardless of this choice. Also, since $\rho$ is a defining function for $\partial \bar{M}$, this quantity is by definition strictly positive at the boundary. We conclude that for any conformally compact metric $g$,

$$
\sigma_{k}\left(A_{g}\right)=\left(-\frac{1}{2}|d \rho| \frac{2}{g}\right)^{k}\left(\begin{array}{c}
n+1 \\
k
\end{array}\right)+\mathcal{O}(\rho),
$$

near $\partial \bar{M}$. If $\sigma_{k}\left(A_{g}\right)$ is constant on $M$, then necessarily $|d \rho| \frac{2}{g}$ is constant along the boundary, and so, multiplying $g$ by a constant, we may as well 
assume that

$$
|d \rho|_{g}^{2} \equiv 1 \quad \text { when } \quad \rho=0 .
$$

In this case, the limit of $\sigma_{k}\left(A_{g}\right)$ at any point of $\partial \bar{M}$ equals the particular constant $(-1)^{k} \beta_{k}^{0}$ which corresponds to the hyperbolic metric $g_{0}$ on $B^{n+1}$, namely

$$
\beta_{k}^{0}:=2^{-k}\left(\begin{array}{c}
n+1 \\
k
\end{array}\right)
$$

Our main result gives a rich class of conformally compact $\sigma_{k}$-Yamabe metrics on the manifold $M$, granting the existence of at least one such metric. In particular, it states that the deformation theory for this problem is always unobstructed whenever $\beta>0$. More precisely, we have:

Theorem 1. Let $\bar{M}$ be a compact smooth manifold with boundary and $\rho$ a fixed defining function for $\partial \bar{M}$. For any metric $\bar{g}$ on $\bar{M}$, denote by $[\bar{g}]$ its conformal class. Suppose that $\sigma_{k}\left(A_{\rho^{-2} \bar{g}}\right)=(-1)^{k} \beta_{k}^{0}$. Then there is a $\mathcal{C}^{2, \alpha}$ neighborhood $\mathcal{U}$ of $[\bar{g}]$ in the space of conformal classes on $\bar{M}$ such that every conformal class $\left[\bar{g}^{\prime}\right]$ in this neighborhood contains a unique metric $\bar{g}_{u}^{\prime}=e^{2 u} \bar{g}^{\prime}$ with

$$
\sigma_{k}\left(A_{\rho^{-2} \bar{g}_{u}^{\prime}}\right)=(-1)^{k} \beta_{k}^{0},
$$

which is near to $\bar{g}$; the set of these solution metrics fills out an (open piece of an) analytic Banach submanifold, with respect to an appropriate Banach topology.

As noted above, the analogue of this theorem holds also when $M$ is compact without boundary, and the proof is similar but even more straightforward. For the record, we state this result too:

Theorem 2. Fix $\beta>0$. Let $g$ be a metric on the compact manifold $M$ and $[g]$ its conformal class. Suppose that $A_{g} \in \Gamma_{k}^{-}($see $\S 1)$ and $\sigma_{k}\left(A_{g}\right)=(-1)^{k} \beta$. Then there is a neighborhood $\mathcal{U}$ of $[g]$ in the space of conformal classes on $M$ such that every conformal class $\left[g^{\prime}\right]$ in this neighborhood contains a unique metric $g_{u}^{\prime}=e^{2 u} g^{\prime}$ with

$$
\sigma_{k}\left(A_{g_{u}^{\prime}}\right)=(-1)^{k} \beta,
$$

which is near to $g$; the set of these solution metrics fills out an (open piece of an) analytic Banach submanifold, with respect to an appropriate Banach topology.

Let us return to conformally compact metrics, and connect Theorem 1 with the first theme discussed in the introduction. To begin with, notice that a metric $g$ is Einstein if and only if:

$$
\operatorname{Ric}_{g}=\frac{R}{n+1} g .
$$


It is well-known that if this is the case then the scalar curvature $R$ is constant. Now (9) is equivalent to either of the two conditions:

$$
\begin{gathered}
A_{g}=\frac{R}{2 n(n+1)} g, \quad \text { or } \\
\sigma_{k}\left(A_{g}\right)=\left(\frac{R}{2 n(n+1)}\right)^{k}\left(\begin{array}{c}
n+1 \\
k
\end{array}\right) \text { for } k=1, \ldots, n+1 .
\end{gathered}
$$

Hence, Poincaré-Einstein metrics are also $\sigma_{k}$-Yamabe metrics for every $k=$ $1, \cdots, n+1$ and, if the scalar curvature is normalized so that $R=-n(n+1)$, the constants $\sigma_{k}\left(A_{g}\right)$ must equal the constants $(-1)^{k} \beta_{k}^{0}$ for hyperbolic space. In particular, the moduli space $\mathcal{E}$ of $\mathrm{P}$-E metrics is equal to the intersection of the $\Sigma_{k}\left(\beta_{k}^{0}\right)$ over all $k$. This gives:

Corollary 1. The moduli space $\mathcal{E}$ of conformally compact Poincaré-Einstein metrics is a finite intersection of Banach submanifolds, and is closed in the space of conformally compact metrics on $M$.

The first statement in this corollary follows directly from the preceding discussion and Theorem 1, while the second statement follows from the fact that the space of $\sigma_{1}$-Yamabe metrics with scalar curvature equal to a fixed negative constant is closed.

In some sense, Corollary 1 shows that the somewhat less tractable space $\mathcal{E}$ is a finite intersection of submanifolds $\Sigma_{j}$, each of which is an analytic submanifold, but more importantly, each of which has an unobstructed deformation theory. This amounts to some sort of figurative 'factorization' of the Einstein equations into $n+1$ scalar (albeit fully nonlinear) equations.

The plan for the rest of this paper is as follows: $\S 1$ reviews the structure of the functionals $\mathcal{F}_{k}$ and their linearizations $\mathcal{L}_{k}$, and this is followed in $\S 2$ by a discussion of the function spaces and of the mapping properties of the $\mathcal{L}_{k}$ on these spaces. The deformation theory for the $\sigma_{k}$-Yamabe equations and the proof of Theorems 1 and 2 is the topic of $\S 3$. Finally, $\S 4$ contains a list of some interesting open questions raised by the results here.

\section{The functionals $\mathcal{F}_{k}$.}

Let us fix a conformally compact metric $g_{0}$, which we may as well take to be smooth, i.e., $g_{0}=\rho^{-2} \bar{g}_{0}$, where both $\rho$ and $\bar{g}_{0}$ are $\mathcal{C}^{\infty}$ on $\bar{M}$. Fix also a constant $\beta>0$. Recall that the metric $g=e^{2 u} g_{0}$ is in $\Sigma_{k}(\beta)$, and so has $\sigma_{k}\left(A_{g}\right)=(-1)^{k} \beta$, provided

$$
\mathcal{F}\left(g_{0}, u, \beta\right)=\sigma_{k}\left(D d u-d u \otimes d u+\frac{1}{2}|d u|^{2} g_{0}-A_{g_{0}}\right)-\beta e^{2 k u}=0 .
$$


In this section we recall some facts about the ellipticity of this operator and the structure of its linearization. These facts are taken from [22], and we refer there for all proofs and further discussion.

To approach the issue of ellipticity, first consider the $k^{\text {th }}$ elementary symmetric function $\sigma_{k}$ as a function on vectors $\lambda=\left(\lambda_{1}, \cdots, \lambda_{n+1}\right) \in \mathbb{R}^{n+1}$. Let $\Gamma_{k}^{+}$denote the connected component of the open set $\left\{\lambda: \sigma_{k}(\lambda)>0\right\}$ containing the positive orthant $\left\{\lambda: \lambda_{j}>0 \forall j\right\}$. These are all convex cones with vertices at the origin and

$$
\left\{\lambda: \lambda_{j}>0 \forall j\right\}=\Gamma_{n+1}^{+} \subset \Gamma_{n}^{+} \subset \cdots \subset \Gamma_{1}^{+}=\left\{\lambda: \sigma_{1}(\lambda)>0\right\} .
$$

Also, let $\Gamma_{k}^{-}=-\Gamma_{k}^{+}$. A real symmetric matrix is said to lie in $\Gamma_{k}^{ \pm}$if its eigenvalues lie in the corresponding set.

We may equally well consider symmetric two-tensors and their eigenvalues relative to a metric $g$, and so we shall transfer to this setting, which is more natural in terms of the geometric notation.

Proposition 1. If $A_{g} \in \Gamma_{k}^{-}$, then $u \rightarrow \mathcal{F}_{k}(g, u, \beta)$ is elliptic at any solution of $\mathcal{F}_{k}(g, u, \beta)=0$.

The proof of this in [22] (see also [21]) relies on the computation of the linearization of $\mathcal{F}_{k}$ in the direction of the conformal factor $u$. The neatest formulation of this requires a definition from linear algebra. For any real, symmetric matrix $B$, and any $q=0, \cdots, n+1$, define the $q^{\text {th }}$ Newton transform of $B$ as the new real, symmetric matrix

$$
T_{q}(B)=\sigma_{q}(B) I-\sigma_{q-1}(B) B+\cdots+(-1)^{q} B^{q} .
$$

Of course, $T_{n+1}(B)=0$. If $B$ is a symmetric two-tensor, then $T_{q}(B)$ is defined as a symmetric two-tensor in the obvious way. Now suppose that $B=B(\varepsilon)$ depends smoothly on a parameter $\varepsilon$, and write $B^{\prime}(0)=\dot{B}$. It is proved in $[\mathbf{2 0}]$ that

$$
\left.\frac{d}{d \varepsilon}\right|_{\varepsilon=0} \sigma_{k}(B(\varepsilon))=\operatorname{Tr}\left(\dot{B} T_{k-1}(B)\right) .
$$

(For symmetric two-tensors, this trace is just the $g$-inner product of $\dot{B}$ with $\left.T_{k-1}(B).\right)$

We apply this to the Schouten tensors associated to the family of metrics $g(\varepsilon)=e^{2 \varepsilon \phi} g$ where $g \in \Sigma_{k}\left(\beta_{k}\right)$. We may use the metric $g$ to identify symmetric 2-tensors with $(n+1) \times(n+1)$ matrices, or completely equivalently, compute traces of such tensors using the metric and regard the trace of the product of matrices on the right side of (10) as the $g$-inner product of tensors. We have

$$
B(\varepsilon)=\left(-A_{g}+\varepsilon D d \phi+\varepsilon^{2}\left(\frac{1}{2}|d \phi|^{2}-d \phi \otimes d \phi\right)\right),
$$


so that $B(0)=B=-A_{g}$ and $\dot{B}(0)=\dot{B}=D d \phi$. This gives the formula

$$
\mathcal{L}_{k} \phi:=\left.D \mathcal{F}_{k}\right|_{g, 0}(0, \phi)=\left\langle T_{k-1}\left(-A_{g}\right), D d \phi\right\rangle_{g}-2 k \beta_{k} \phi .
$$

The proof of Proposition 1 in general (i.e., when $g$ is not necessarily a solution itself and when the linearization is computed at some solution $u \neq 0$ ) relies on the (nonobvious) fact that $T_{k-1}(B)$ is positive definite when $B \in \Gamma_{k}^{+}$. We refer to [22] (or [21]) for further details.

Let us compute $\mathcal{L}_{k}$ more explicitly when $g$ is hyperbolic, or in fact, when $g$ is an arbitrary Poincaré-Einstein metric. We shall always normalize the metric so that the scalar curvature is given by $R=-n(n+1)$, in which case the Einstein condition becomes Ric $=-n g$ and $\sigma_{k}\left(A_{g}\right)=(-1)^{k} \beta_{k}^{0}$. By (9), if $g$ is P-E then $A_{g}=-\frac{1}{2} g$, and so

$$
T_{k-1}\left(-A_{g}\right)=2^{1-k} T_{k-1}(g)=2^{1-k} \sum_{j=0}^{k-1}(-1)^{j}\left(\begin{array}{c}
n+1 \\
k-1-j
\end{array}\right) g .
$$

One may check by induction that this sum has a closed form expression, and this leads to the identity

$$
T_{k-1}\left(-A_{g}\right)=c_{k, n} g, \quad \text { where } \quad c_{k, n}=2^{1-k}\left(\begin{array}{l}
n \\
k
\end{array}\right) .
$$

The key observation here is that $c_{k, n}>0$. Altogether, we obtain the formula

$$
\mathcal{L}_{k} \phi=c_{k, n} \Delta \phi-2 k \beta_{k}^{0} \phi,
$$

which holds whenever $g$ is Poincaré-Einstein with Ric $=-n g$.

If $g \in \Sigma_{k}(\beta)$ is a more general solution (i.e., not necessarily P-E), then $\mathcal{L}_{k}$ is more complicated. However, certain properties remain valid. A direct calculation yields:

Proposition 2. Suppose $g \in \Sigma_{k}(\beta), \beta>0$, and let $\mathcal{L}_{k}$ denote the linearization of $\mathcal{F}_{k}$ at $u=0$. Then

$$
\mathcal{L}_{k} \phi=c_{k, n} \Delta_{g} \phi-2 k \beta \phi+\rho^{3} E \phi,
$$

where $E$ is a second order operator with bounded coefficients on $\bar{M}$ (smooth if $\rho$ and $\bar{g}=\rho^{2} g$ are smooth), and without constant term.

Note especially one of the key point in this result that the operator $E$ contains no constant term.

We remark that (13) may also be obtained from general principles involving the theory of uniformly degenerate operators $[\mathbf{1 5}]$ and [16]. Since some of the main results of this theory will be invoked later anyway, we digress briefly to explain this setup. Choose coordinates $(x, y):=\left(x, y_{1}, \ldots, y_{n}\right)$, 
$x \geq 0$ near a point of the boundary of $\bar{M}$. A second order operator $L$ is said to be uniformly degenerate if it may be expressed in the form

$$
L=\sum_{j+|\alpha| \leq 2} a_{j, \alpha}(x, y)\left(x \partial_{x}\right)^{j}\left(x \partial_{y}\right)^{\alpha} .
$$

The coefficients may be scalar or matrix-valued, and although we usually assume they are smooth, it is easy to extend most of the main conclusions of this theory when they are polyhomogeneous, or of some finite regularity. Operators of this type arise naturally in geometry, and in particular all of the natural geometric operators associated to a conformally compact metric are uniformly degenerate. Note that the error term $\rho^{3} E$ in (13) is actually of the form $\rho E^{\prime}$ where $E^{\prime}$ is some second order uniformly degenerate operator without constant term.

The 'uniformly degenerate symbol' of this operator is elliptic provided

$$
\sigma(L)(x, y ; \xi, \eta):=\sum_{j+|\alpha|=2} a_{j, \alpha}(x, y) \xi^{j} \eta^{\alpha} \neq 0 \quad \text { when } \quad(\xi, \eta) \neq 0 .
$$

(For systems, we require $\sigma(L)$ to be invertible as a matrix when $(\xi, \eta) \neq 0$.) We also define the associated normal operator

$$
N(L):=\sum_{j+|\alpha| \leq 2} a_{j, \alpha}(0, y)\left(s \partial_{s}\right)^{j}\left(s \partial_{v}\right)^{\alpha} .
$$

The boundary variable $y$ enters only as a parameter, while the 'active' variables $(s, v)$ in this expression may be regarded as formal, but in fact are naturally identified with linear coordinates on the inward pointing half-tangent space $T_{(0, y)}^{+} M$. In particular:

Proposition 3. If $g$ is a smooth conformally compact metric (normalized so that $|d \rho| \frac{2}{g}=1$ at $\left.\partial \bar{M}\right)$, then its Laplace-Beltrami operator $\Delta_{g}$ is an elliptic uniformly degenerate operator with normal operator

$$
N\left(\Delta_{g}\right)=\Delta_{\mathbb{H}^{n+1}}:=\left(s \partial_{s}\right)^{2}+s^{2} \Delta_{\mathbb{R}^{n}}-n s \partial_{s} .
$$

Furthermore, if $g \in \Sigma_{k}\left(\beta_{k}^{0}\right)$, then the linearization $\mathcal{L}_{k}$ of $\mathcal{F}_{k}$ at $u=0$ is also elliptic and uniformly degenerate, with normal operator

$$
\mathcal{L}_{k}^{0}:=N\left(\mathcal{L}_{k}\right)=c_{k, n}\left(\left(s \partial_{s}\right)^{2}+s^{2} \Delta_{\mathbb{R}^{n}}-n s \partial_{s}\right)-2 k \beta_{k}^{0} .
$$

As we explain in the next section, the operator $\mathcal{L}_{k}$ is Fredholm on various natural function spaces. This specializes a criterion which is applicable to other more general uniformly degenerate operators $L$, namely that $L$ is Fredholm if and only if two separate ellipticity conditions hold: First, the symbol $\sigma(L)$ should be invertible, and in addition, the normal operator $N(L)$ must be invertible on certain weighted $L^{2}$ spaces. 


\section{Function spaces and mapping properties.}

Let $\mathcal{L}_{k}$ be the linearization considered in the last section. We shall now describe some of its mapping properties. As indicated above, these properties also hold for more general elliptic, uniformly degenerate operators $L$.

We first review one particular scale of function spaces which is convenient in the present setting, and then state the mapping properties on them enjoyed by $\mathcal{L}_{k}$. The material here is taken from [15], to which we refer for further discussion and proofs.

Fix a reference (smooth) conformally compact metric $g_{0}=\rho^{-2} \bar{g}_{0}$; also, choose a smooth boundary coordinate chart $(x, y)$ as in the previous section, and recall the basic vector fields $x \partial_{x}$ and $x \partial_{y_{j}}, j=1, \ldots, n$. Since $x$ is a smooth nonvanishing multiple of $\rho$ near $\partial \bar{M}$, these vector fields are all of uniformly bounded lengths with respect to $g_{0}$, and are also uniformly independent as $x \searrow 0$. There are two equivalent ways to define the Hölder space $\Lambda^{\ell, \alpha}(M), \ell \in \mathbb{N}, \alpha \in(0,1)$. In either case, it suffices to work in a boundary coordinate chart. The first is to set

$$
\Lambda^{0, \alpha}(M):=\left\{u: \sup \frac{\left|u(x, y)-u\left(x^{\prime}, y^{\prime}\right)\right|\left(x+x^{\prime}\right)^{\alpha}}{\left|x-x^{\prime}\right|^{\alpha}+\left|y-y^{\prime}\right|^{\alpha}}\right\},
$$

where the supremum is taken first over all points $w=(x, y), w^{\prime}=\left(x^{\prime}, y^{\prime}\right)$, $w \neq w^{\prime}$, which lie in some coordinate cube $B$ centered at a point $w_{0}=$ $\left(x_{0}, y_{0}\right)$ of sidelength $\frac{1}{2} x_{0}$, and then over all such cubes. The other is to let $B$ denote a ball of unit radius with respect to the metric $g_{0}$ centered at $w_{0}$, and to replace the quotient in this definition by

$$
\frac{\left|u(x, y)-u\left(x^{\prime}, y^{\prime}\right)\right|}{\operatorname{dist}_{g_{0}}\left(w, w^{\prime}\right)^{\alpha}}
$$

and then take the supremum over all $w \neq w^{\prime} \in B$, and then over all such balls $B$.

This latter definition is more geometric, while the former definition clearly implies the scale invariance of these spaces, namely that if $u(w)$ is defined (and, say, compactly supported) in one of these coordinate charts and if we define $u_{\varepsilon}(w)=u(w / \varepsilon)$, then the associated norms of $u$ and $u_{\varepsilon}$ are the same.

We shall also use a few other closely related spaces:

- For $\ell \in \mathbb{N}$ and $\alpha \in(0,1)$, let

$$
\Lambda^{\ell, \alpha}(M):=\left\{u:\left(x \partial_{x}\right)^{j}\left(x \partial_{y}\right)^{\beta} u \in \Lambda^{0, \alpha}(M) \forall j+|\beta| \leq \ell\right\} .
$$

- For $\gamma \in \mathbb{R}, \ell \in \mathbb{N}$ and $\alpha \in(0,1)$, let

$$
\rho^{\gamma} \Lambda^{\ell, \alpha}(M):=\left\{u: u=\rho^{\gamma} \widetilde{u}, \quad \text { where } \quad \widetilde{u} \in \Lambda^{\ell, \alpha}(M)\right\} .
$$


Thus the first of these are the natural higher order Hölder spaces associated to the geometry of $g_{0}$, or equivalently, to differentiations with respect to the vector fields $x \partial_{x}$ and $x \partial_{y}$. The second of these spaces are the usual weighted analogues. The corresponding norms are $\|\cdot\|_{\ell, \alpha}$ and $\|\cdot\|_{\ell, \alpha, \gamma}$, respectively.

We could equally easily have defined $L^{2}$ - and $L^{p}$-based Sobolev spaces, corresponding to differentiations with respect to the vector fields $x \partial_{x}$ and $x \partial_{y}$. The mapping properties we state below all have direct analogues for these spaces. However, as usual, Hölder spaces are perhaps the simplest to deal with for nonlinear PDE.

Now let us turn to the mapping properties of $\mathcal{L}_{k}$ in the case where the conformally compact metric $g$ at which $\mathcal{L}_{k}$ is computed satisfies $\sigma_{k}\left(A_{g}\right)=$ $(-1)^{k} \beta_{0}^{k}$. First of all, it follows immediately from the definitions that

$$
\mathcal{L}_{k}: \rho^{\gamma} \Lambda^{\ell+2, \alpha}(M) \longrightarrow \rho^{\gamma} \Lambda^{\ell, \alpha}(M)
$$

is a bounded mapping for any $\gamma \in \mathbb{R}$ and $0 \leq \ell$. However, this map is not well-behaved for many values of the weight parameter $\gamma$. There are two ways this may occur. First if $\gamma$ is sufficiently large positive, then it is not hard to see that (17) has an infinite dimensional cokernel, while dually, if $\gamma$ is sufficiently large negative, then (17) has an infinite dimensional nullspace. Although we do not use it here, less trivial is the fact that in either of these two cases the mapping is semi-Fredholm (i.e., has closed range and either the kernel or cokernel are finite-dimensional).

However, for certain values of $\gamma$ the range of this mapping may not be closed. This is determined by a consideration of the indicial roots of $\mathcal{L}_{k}$. We say that $\gamma$ is an indicial root of $\mathcal{L}_{k}$ if $\mathcal{L}_{k}\left(\rho^{\gamma}\right)=O\left(\rho^{\gamma+1}\right)$ (note that because of the uniform degeneracy of $\mathcal{L}_{k}, \mathcal{L}_{k}\left(\rho^{\gamma}\right)=O\left(\rho^{\gamma}\right)$ is true for any value of $\gamma)$. Thus $\gamma$ is an indicial root only if some special cancellation occurs. It is clear that the indicial roots of $\mathcal{L}_{k}$ agree with those of its normal operator $\mathcal{L}_{k}^{0}$, and then (16) shows that $\gamma$ is an indicial root if and only if

$$
c_{k, n}\left(\gamma^{2}-n \gamma\right)-2 k \beta_{k}^{0}=0,
$$

or in other words $\gamma \in\left\{\gamma_{ \pm}\right\}$where

$$
\gamma_{ \pm}:=\frac{n}{2} \pm \sqrt{\frac{n^{2}}{4}+\frac{2 k \beta_{k}^{0}}{c_{k, n}}} .
$$

In particular

$$
\gamma_{-}<0<n<\gamma_{+}
$$

since $\beta_{k}, c_{k, n}>0$. 
The relevance of these indicial roots to the mapping properties of (17) is that when $\gamma$ is equal to one of these two values, then (17) does not have closed range. At heart, this stems from the fact that the equation

$$
\mathcal{L}_{k}^{0} u=s^{\gamma_{ \pm}}
$$

has solution $u=c s^{\gamma_{ \pm}}(\log s)$ for some constant $c$, i.e., the inhomogeneous term is in the appropriate weighted Hölder space but the solution $u$ just misses being in this space.

Despite these cautions, we have the following basic result:

Mapping properties: If $\gamma_{-}<\gamma<\gamma_{+}$, then the mapping (17) is Fredholm of index zero.

The main result of [15] is a considerably more general theorem of this sort for more general elliptic uniformly degenerate differential operators. There are two special features of $\mathcal{L}_{k}$ which enter into the precise form of the statement here. First, there is a nontrivial interval $\left(\gamma_{-}, \gamma_{+}\right)$between the two indicial roots $\gamma_{ \pm}$, allowing for the possibility of a 'Fredholm range'. Second, the Fredholm index is zero for $\gamma$ in this interval ultimately because $\mathcal{L}_{k}$ is self-adjoint on $L^{2}\left(d V_{g}\right)$.

We claim that the mapping (17) is actually invertible when $\gamma$ is in this Fredholm range. By the result just stated, this claim will be proved if we show that when $\gamma \in\left(\gamma^{-}, \gamma^{+}\right)$the nullspace of $\mathcal{L}_{k}$ is trivial. We do this now. The basic observation is that the constant term in $\mathcal{L}_{k}$ is negative. If $\gamma>0$, then any $\phi \in \rho^{\gamma} \Lambda^{\ell+2, \alpha}$ vanishes at $\partial \bar{M}$, and thus if $\mathcal{L}_{k} \phi=0$, the maximum principle implies that $\phi=0$, as desired. To prove the claim for every $\gamma \in\left(\gamma_{-}, \gamma_{+}\right)$, we first compute that for any two values $\gamma^{\prime}, \gamma^{\prime \prime} \in\left(\gamma_{-}, \gamma_{+}\right)$ and constants $c^{\prime}, c^{\prime \prime}>0$ the function

$$
w_{c^{\prime}, c^{\prime \prime}}:=c^{\prime} \rho^{\gamma^{\prime}}+c^{\prime \prime} \rho^{\gamma^{\prime \prime}}
$$

satisfies $\mathcal{L}_{k} w_{c, c^{\prime}}<0$ in some small collar neighbourhood $\bar{M}_{\tau}$ of the boundary, where $0 \leq \rho \leq \tau$. Now fix $\gamma^{\prime \prime} \in\left(\gamma, \gamma_{+}\right)$and choose $c^{\prime \prime}$ so that $|\phi| \leq c^{\prime \prime} \rho^{\gamma^{\prime \prime}}$ on the set where $\rho=\tau$. Fixing $\gamma^{\prime} \in\left(\gamma_{-}, \gamma\right)$ and $c^{\prime}>0$, then for any $\varepsilon>0$, the functions $v_{ \pm}:=w_{c^{\prime}, c^{\prime \prime}} \pm \phi$ satisfy $\mathcal{L}_{k} v_{ \pm}<0$ in $\bar{M}_{\tau}$ and $v_{ \pm} \geq 0$ both at the inner boundary $\rho=\tau$ and also at near the outer boundary where $\rho=0$. By the maximum principle again, $v_{ \pm}>0$ in $\bar{M}_{\tau}$. Letting $c^{\prime} \searrow 0$, we conclude that $|\phi| \leq c^{\prime \prime} \rho^{\gamma^{\prime \prime}}$. Since we may choose $\gamma^{\prime \prime}>0$, this implies that $\phi$ is bounded in $M$ and vanishes at $\partial \bar{M}$. This reduces us to the previous case.

This reasoning shows that, if $\gamma \in\left(\gamma_{-}, \gamma_{+}\right)$and $\phi \in \rho^{\gamma} \Lambda^{\ell+2, \alpha}$ is a solution of $\mathcal{L}_{k} \phi=0$, then $\phi \in \rho^{\gamma^{\prime}} \Lambda^{\ell+2, \alpha}$ for any $\gamma^{\prime}<\gamma_{+}$. However, on account of the following basic result from [15], a much sharper result is true. 
Regularity of solutions: If $\gamma_{-}<\gamma<\gamma_{+}$and $\phi \in \rho^{\gamma} \Lambda^{\ell+2, \alpha}$ is a solution of $\mathcal{L}_{k} \phi=f$ where $f$ vanishes to all orders at $\partial \bar{M}$, then as $x \rightarrow 0$,

$$
\phi(x, y) \sim \sum_{j=0}^{\infty} \phi_{j}(y) x^{\gamma_{+}+j}, \quad \text { with } \quad \phi_{j}(y) \in \mathcal{C}^{\infty}(\partial \bar{M}),
$$

in particular $\phi \in \rho^{\gamma+\mathcal{C}^{\infty}}(\bar{M})$.

\section{Perturbation theory in $\Sigma_{k}$.}

We now proceed to the main deformation result. Let $\bar{M}$ be a smooth compact, $n+1$ dimensional manifold with boundary. We fix a smooth defining function $\rho$ for $\partial \bar{M}$. For any $\ell \in \mathbb{N}$ and any $\alpha \in(0,1)$ we define

$$
\mathfrak{M}^{\ell, \alpha}(\bar{M}):=\left\{\bar{g} \in \mathcal{C}^{\ell, \alpha}\left(\bar{M} ; S^{2}(\bar{M})\right): \quad|d \rho|_{\bar{g}}^{2}=1 \quad \text { on } \quad \partial \bar{M}\right\} .
$$

Having set things up carefully, the proof of Theorem 1 is almost immediate. Let $g \in \Sigma_{k}\left(\beta_{k}^{0}\right)$ and consider the mapping

$$
\mathcal{H}: \mathfrak{M}^{2, \alpha}(\bar{M}) \times \rho^{\gamma} \Lambda^{2, \alpha}(M) \longrightarrow \rho^{\gamma} \Lambda^{0, \alpha}
$$

defined by

$$
\mathcal{H}(h, u):=\mathcal{F}_{k}\left(\rho^{-2} h, u, \beta_{k}^{0}\right) .
$$

Near $g$, the set $\Sigma_{k}\left(\beta_{k}^{0}\right)$ is identified with the zero set of $\mathcal{H}$. In particular, $(h, u)=(g, 0) \in \mathcal{H}^{-1}(0)$.

To find all other nearby solutions, we shall apply the implicit function theorem, very much in the spirit of the closely related papers [18] and [11]. Thus we must check two things:

(i) The mapping $\mathcal{H}$ in (18) is a smooth mapping between the corresponding Banach spaces.

(ii) The linear map $\left.u \longrightarrow D \mathcal{H}\right|_{g, 0}(0, u)$ is surjective between these spaces. The first of these is straightforward from the definitions and (2) and (7), provided we choose the weight parameter $\gamma \in(0,1)$. As for the other, recall that the restriction of this Fréchet derivative to tangent vectors of the form $(0, u)$ corresponds to the operator $\mathcal{L}_{k}$. We have already checked that this is surjective provided we choose the weight parameter $\gamma \in\left(\gamma_{-}, \gamma_{+}\right)$. But since $(0,1) \subset\left(\gamma_{-}, \gamma_{+}\right)$, these restrictions on $\gamma$ are not inconsistent. Thus fixing $\gamma \in(0,1)$, we obtain a smooth mapping

$$
\Phi: \mathfrak{M}^{2, \alpha}(\bar{M}) \longrightarrow \rho^{\gamma} \Lambda^{2, \alpha}(M)
$$

with $\Phi(g)=0$ and such that

$$
\mathcal{H}(h, \Phi(h)) \equiv 0 .
$$

Furthermore, all solutions of $\mathcal{H}(h, u)$ in a sufficiently small neighborhood of $(g, 0)$ are of this form. This concludes the proof of Theorem 1. 
We omit the proof of Theorem 2 because it is nearly identical; indeed, the only difference is that standard elliptic theory replaces the Fredholm theory for uniformly degenerate operators we have quoted.

\section{Open questions and further directions.}

We conclude this note by raising a few other problems and questions related to the results and methods here.

a) Because of the difficulty in obtaining a $\mathcal{C}^{2}$ estimate for the $\sigma_{k}$-Yamabe problem when $k>1$, it is worth wondering whether it might be worthwhile to pose a weaker version of this problem, at least for conformally compact metrics on manifolds with boundary: Namely, given a conformal class $\left[h_{0}\right]$ on $\partial \bar{M}$, is it possible to extend this conformal class to at least some conformal class $[\bar{g}]$ on the interior such that the $\sigma_{k}$-Yamabe problem is solvable in $[\bar{g}]$ ? Probably there are infinitely many such extensions, as is the case when $k=1$, but the added flexibility in this formulation may be of some use.

b) We have shown in Theorem 1 that $\Sigma_{k}\left(\beta_{k}^{0}\right)$ is a Banach submanifold in a neighborhood of $g$, and furthermore that it may be regarded as a graph over the space of conformal classes, or at least those conformal classes near to $g$. For $k=1$, every conformal class on $\bar{M}$ contains a unique representative lying in $\Sigma_{1}\left(\beta_{1}^{0}\right)$, and thus $\Sigma_{1}\left(\beta_{1}^{0}\right)$ is a graph globally over the space $\mathfrak{C}$ of all conformal classes. It is not known whether this remains true when $k>1$, and thus we define

$$
\mathfrak{C}_{k}=\left\{\mathfrak{c} \in \mathfrak{C}: \mathfrak{c} \text { contains at least one } g \in \Sigma_{k}\left(\beta_{k}^{0}\right)\right\} .
$$

Note that $\mathfrak{C}_{1}=\mathfrak{C}$, and Theorem 1 shows that $\mathfrak{C}_{k}$ is open in $\mathfrak{C}$ for every $k$.

It seems central to understand whether $\mathfrak{C}_{k}=\mathfrak{C}$, or in other words, whether every conformal class on $M$ contains a conformally compact $\sigma_{k}$-Yamabe metric. Related to this is the observation that we do not know whether each of the submanifolds $\Sigma_{k}$ itself is closed; this depends ultimately on whether some version of this $\mathcal{C}^{2}$ estimate holds.

It also seems interesting to ask for which $\ell$-tuple $J=\left\{j_{1}, \ldots, j_{\ell}\right\} \subset$ $\{1, \ldots, n+1\}$ is the set

$$
\Sigma_{J}:=\Sigma_{j_{1}}\left(\beta_{j_{1}}^{0}\right) \cap \cdots \cap \Sigma_{j_{\ell}}\left(\beta_{j_{\ell}}^{0}\right),
$$

closed. Notice that if $1 \in J$, then this is certainly true because the $\mathcal{C}^{2}$ estimate for the conformal factor is routine for the scalar curvature equation.

c) The regularity of the metrics $g \in \Sigma_{k}\left(\beta_{k}^{0}\right)$ is an interesting question. When $k=1$ this is resolved in [16], cf. also [19]: If $g$ is a smooth conformally compact metric, then the conformal factor $u$ corresponding to 
the unique solution $\widetilde{g}=e^{2 u} g \in[g]$ has a polyhomogeneous expansion. Presumably a similar result holds for all $k$. Note that unless $\gamma_{+} \in \mathbb{N}$, this expansion will involve nonintegral powers of $\rho$; this should not be viewed negatively, since functions with expansions of this form may be manipulated just as easily as smooth functions.

d) The $\sigma_{k}$-Yamabe problem considered here extends naturally to the more general setting of the singular $\sigma_{k}$-Yamabe problem: Given a smooth metric $g_{0}$ on a compact manifold $M$ and a closed subset $\Lambda \subset M$, when is it possible to find a conformally related metric $g=e^{2 u} g_{0}$ which is both complete on $M \subset \Lambda$ and a $\sigma_{k}$-Yamabe metric? When $k=1$ it is known that the dimension of $\Lambda$ is intimately related to the sign of the imposed scalar curvature of the solution, and very good existence results are known when $\Lambda$ is a submanifold [5] and [17]. What is the correct statement, and to what extent is this true when $k>1$ ? There are a number of interesting analytic problems of this nature, and we shall return to this soon.

e) In general (not just in the conformally compact setting), $\mathcal{E}$ sits inside the finite intersection $\cap \Sigma_{k}$. Does it appear here as a finite codimensional analytic set, and if so, is this related to some sort of Kuranishi reduction for the perturbation theory for $\mathcal{E}$ ?

f) If $g$ is a conformally compact metric on $M$ and if $\sigma_{k}\left(A_{g}\right)=(-1)^{k} \beta$ and $\sigma_{k^{\prime}}\left(A_{g}\right)=(-1)^{k^{\prime}} \beta^{\prime}$ are constant, then necessarily

$$
\left(\frac{\beta}{\beta_{k}^{0}}\right)^{1 / k}=\left(\frac{\beta^{\prime}}{\beta_{k^{\prime}}^{0}}\right)^{1 / k^{\prime}} .
$$

This follows at once from (6) and (7).

For any $(n+1)$-tuple of numbers $\beta \equiv\left(\beta_{1}, \ldots, \beta_{n+1}\right)$, define

$$
\Sigma(\beta):=\bigcap_{k=1}^{n+1} \Sigma_{k}\left(\beta_{k}\right) .
$$

By (20) it is clear that in the class of conformally compact metrics, $\Sigma(\beta)=\emptyset$ unless $\beta_{k}=(-\lambda)^{k} \beta_{k}^{0}$ for some $\lambda>0$ and for all $k$.

By contrast, on any compact manifold without boundary, it may happen that for some other $(n+1)$-tuple $\beta$ of positive numbers, the set $\Sigma(\beta)$ is nonempty. If $g \in \Sigma(\beta)$, then $\operatorname{Ric}_{g}$ has constant eigenvalues. In particular, metrics with $\nabla$ Ric $=0$ are in $\Sigma(\beta)$ for some $\beta$. However, the reverse inclusion may not be true and it appears that very little is known about metrics with Ricci tensor having constant eigenvalues, but cf. [4].

The (presumably) smaller class of metrics with parallel Ricci tensor is more tractable, but it does not seem to be known if the eigenvalues 
can be constant without the Ricci tensor being parallel. Examples would be very welcome. Also, in any setting (compact or conformally compact or ...) it seems to be a very basic problem in Riemannian geometry to ask what are the possible $(n+1)$-tuples $\left(\beta_{1}, \ldots, \beta_{n+1}\right)$ for which $\Sigma_{1}\left(\beta_{1}\right) \cap \cdots \cap \Sigma_{n+1}\left(\beta_{n+1}\right)$ is nonempty?

Acknowledgements. We wish to thank Paul Yang for providing initial inspiration for these results in pointing out the dearth of examples of $\sigma_{2^{-}}$ Yamabe metrics in the negative case, and suggesting some possible lines of enquiry. Matt Gursky's encouragement was also quite helpful.

\section{References}

[1] M. Anderson, $L^{2}$ curvature and volume renormalization for AHE metrics on 4-manifolds, Math. Research Letters, 8 (2001), 171-188, MR 2002k:53079, Zbl 0999.53034.

[2] _ Boundary regularity, uniqueness and non-uniqueness for AH Einstein metrics on 4-manifolds, to appear in Advances in Math., math-DG/0104171.

[3] _ Einstein metrics with prescribed conformal infinity on 4-manifolds, mathDG/0105243.

[4] V. Apostolov, T. Draghici and A. Moroianu, A splitting theorem for Kähler manifolds whose Ricci tensors have constant eigenvalues, Internat. J. Math., 12 (2001), 769-789. MR 2002j:53094.

[5] P. Aviles and R. McOwen, Complete conformal metrics with negative scalar curvature in compact Riemannian manifolds, Duke Math. J., 56(2) (1988), 395-398, MR 89b:58224, Zbl 0645.53023.

[6] A. Besse, Einstein Manifolds, Ergebnisse der Mathematik und ihrer Grenzgebiete, 10, Springer-Verlag, Berlin, 1987, MR 88f:53087, Zbl 0613.530.

[7] O. Biquard, Métriques d'Einstein asymptotiquement symmétriques, Astérisque, 265, 2000, MR 2001k:53079, Zbl 0967.53030.

[8] A. Chang, M. Gursky and P. Yang, An equation of Monge-Ampere type in conformal geometry and four-manifolds of positive Ricci curvature, Ann. of Math., 155 (2002), 709-787, MR 2003j:53048.

[9] C. Fefferman and C.R. Graham, Conformal invariants, in 'Elie Cartan et les Mathematiques d'Aujourd'hui', Astérisque, 1985, 95-116, MR 87g:53060, Zbl 0602.53007.

[10] C.R. Graham, Volume and area renormalization for conformally compact Einstein manifolds, in "Proceedings of the 19th Winter School "Geometry and Physics" (Srni, 1999)', Rend. Circ. Mat. Palermo, 2(63) (2000), 31-42, MR 2002c:53073, Zbl 0984.53020.

[11] C.R. Graham and J. Lee, Einstein metrics with prescribed conformal infinity on the ball, Adv. in Math., 87 (1991), 186-225, MR 92i:53041, Zbl 0765.53034.

[12] S. Hawking and D.N. Page, Thermodynamics of black holes in anti-de Sitter space, Comm. Math. Phys., 87 (1983), 577-588, MR 84d:83024.

[13] J. Lee, Fredholm operators and Einstein metrics on conformally compact manifolds, math-DG/0105046. 
[14] J. Lee and T. Parker, The Yamabe problem, Bull. Amer. Math. Soc. (N.S.), 17(1) (1987), 37-91, MR 88f:53001, Zbl 0633.53062.

[15] R. Mazzeo, Elliptic theory of differential edge operators I, Comm. Partial Differential Equations, 16 (1991), 1615-1664, MR 93d:58152, Zbl 0745.58045.

[16] _ Regularity for the singular Yamabe problem, Indiana Univ. Math. J., 40 (1991), 1277-1299, MR 92k:53071, Zbl 0770.53032.

[17] R. Mazzeo and F. Pacard, A construction of singular solutions for a semilinear elliptic equation using asymptotic analysis, J. Differential Geom., 44(2) (1996), 331-370, MR 98a:35040, Zbl 0869.35040.

[18] R. Mazzeo and N. Smale, Conformally flat metrics of constant positive scalar curvature on subdomains of the sphere, J. Differential Geom., 34(3) (1991), 581-621, MR 92i:53034, Zbl 0759.53029.

[19] R. Mazzeo and M. Taylor, Curvature and uniformization, Israel J. Math., 130 (2002), 323-346, CMP 1919 383, Zbl 1003.30031.

[20] R. Reilly, Variational properties of functions of the mean curvatures for hypersurfaces in space forms, J. Differential Geom., 8 (1973), 465-477, MR 49 \#6102, Zbl 0277.53030.

[21] H. Rosenberg, Hypersurfaces of constant curvature in space forms, Bull. Sci. Math., 117(2) (1993), 211-239, MR 94b:53097, Zbl 0787.53046.

[22] J. Viaclovsky, Estimates and some existence results for some fully nonlinear elliptic equations on Riemannian manifolds, Commun. Anal. Geom., 10 (2002), 815-846.

[23] X. Wang, On the Geometry of Conformally Compact Einstein Manifolds, Thesis, Stanford University, 2001.

[24] E. Witten, Anti de Sitter space and holography, Adv. Theor. Math. Phys., 2 (1998), 253-291, hep-th/9802150, MR 99e:81204c, Zbl 0914.53048.

Received May 8, 2002 and revised June 11, 2002. The first author was supported by the NSF under Grant DMS-9971975 and at MSRI by NSF grant DMS-9701755. The second author was supported at MSRI by NSF grant DMS-9701755.

StANFord University

STANFORD CA 94305-2125

E-mail address: mazzeo@math.stanford.edu

Université PARIS XII

FRANCE

E-mail address: pacard@univ-paris12.fr 